\title{
TOWARDS THE DIGITAL PLATFORM AND SMART SERVICES FOR MANAGING SPACE TRAFFIC
}

\author{
PETR O. SKOBELEV \& OLEG I. LAKHIN \\ Institute for Control of Complex Systems of the Russian Academy of Sciences, \\ Software Engineering Company "Smart Solutions", Russia.
}

\begin{abstract}
The objective of the paper is to discuss the increasing complexity of modern space traffic in the nearEarth space and outline the new approach for solving the problem. The requirements and functionality of digital platform for traffic management are presented and examples of problem solving are given. The developed approach will create new opportunities for managing space traffic and resources of the mission control centers for a large number of spacecrafts. Possible approaches to description of spacecraft flights are given. Methods and tools for optimizing the use of ground control complexes to manage large-scale orbital groups have been discussed. Creation of the digital platform and eco-system of smart services for space traffic management will solve the most important problem of space traffic management to increase the effectiveness of the created satellites groups and to protect the spacecrafts from space waste and debris.

Keywords: Digital Platforms, Ground Stations, Management of Conflicts, Networks of Services, Planning and Control, Real Time, Satellites, Space Traffic, Spacecrafts.
\end{abstract}

\section{INTRODUCTION}

Currently, mankind is beginning to perceive outer space not only as an object of research and knowledge acquisition, but also as a new environment, the development of which brings commercial benefits [1]. It is already difficult to imagine life of modern human society without satellite-based navigation, mobile communication, digital maps compiled on the basis of space remote sensing of the Earth, etc.

Modern development of space systems and spacecrafts requires taking into account the following new factors:

- high speed of development of electronics and computer technology;

- development of additive technologies (3D printing) for design and production of spacecraft elements and systems;

- creation of terrestrial computing systems of high power and high reliability;

- development of reliable software used in space activities;

- extensive experience in flight control and operation of spacecrafts and spacecraft systems;

- significant progress in applied sciences sustaining space activities - ballistics, navigation, information processing, management of complex systems, etc.

In the nearest future we can expect the following important stages in development of space activities [2]:

- creation of a large number of cheap and relatively reliable spacecrafts, which have a small mass, small size and significant processing power;

- emergence of autonomous spacecrafts, in which most of the control tasks are carried out on board the spacecraft itself, and interaction with the ground control loop 
is transferred into the area of determining long-term plans for operation of the spacecraft;

- emergence of multi-satellite systems, managed by the principle of "smart swarms";

- "explosive" increase in the number of simultaneously functioning spacecrafts in certain orbits or in a certain area of outer space;

- significant increase in the number of "space waste and debris" due to high intensity of space activities (broken-down spacecrafts, or those which have run out their operation term, unutilized remains of launch vehicles, fragments of collided spacecrafts, etc.);

- increase in the number of participants in space activities due to inclusion of new countries and new commercial companies, etc.

Thus, the boundless (as it seemed before) space, especially the near-Earth space, begins to become very "crowded", which can cause conflicts and reduce the effectiveness of space activities.

Currently the world community at the UN site starts initiative to develop basic rules for joint operations in outer space.

In this paper, we describe the proposed approach to developing space traffic control platform and eco-system of services, which is designed to allow adaptive and coordinated planning and control of spacecraft traffic in real time.

First of all, it is necessary to discuss the current state of the near-Earth space and those conditions in which space systems will operate in the near future.

\section{INCREASING COMPLEXITY OF TRAFFIC MANAGEMENT IN THE NEAR-EARTH SPACE: "SPACE WASTE AND DEBRIS”, EXPLOSIVE INCREASE IN THE COMPOSITION OF SATELLITE GROUPS, EMERGENCE OF NUCLEAR POWER}

At present, many countries are forced to deploy their own control centers for the following objects in the near-Earth space:

- operating spacecrafts;

- upper stages of launch vehicles;

- spacecrafts that have stopped their active existence;

- old satellites, fragments of space vehicles and spent rocket stages, the fragments from their disintegration, erosion and collisions.

In addition, nowadays there is a large number of unmonitored or difficult-to-track objects in space:

- hundreds of thousands of space objects in the size range from 1 to $10 \mathrm{~cm}$;

- hundreds of millions of space objects in the size range from $1 \mathrm{~mm}$ to $1 \mathrm{~cm}$;

- billions of space objects less than $1 \mathrm{~mm}$ in size.

National centers, in their turn, assume availability of specialized micro-control centers for each spacecraft.

However, in case of any problem situation, strong coordination of actions between the teams of such centers is required. For example, the recent situation with a high probability of 


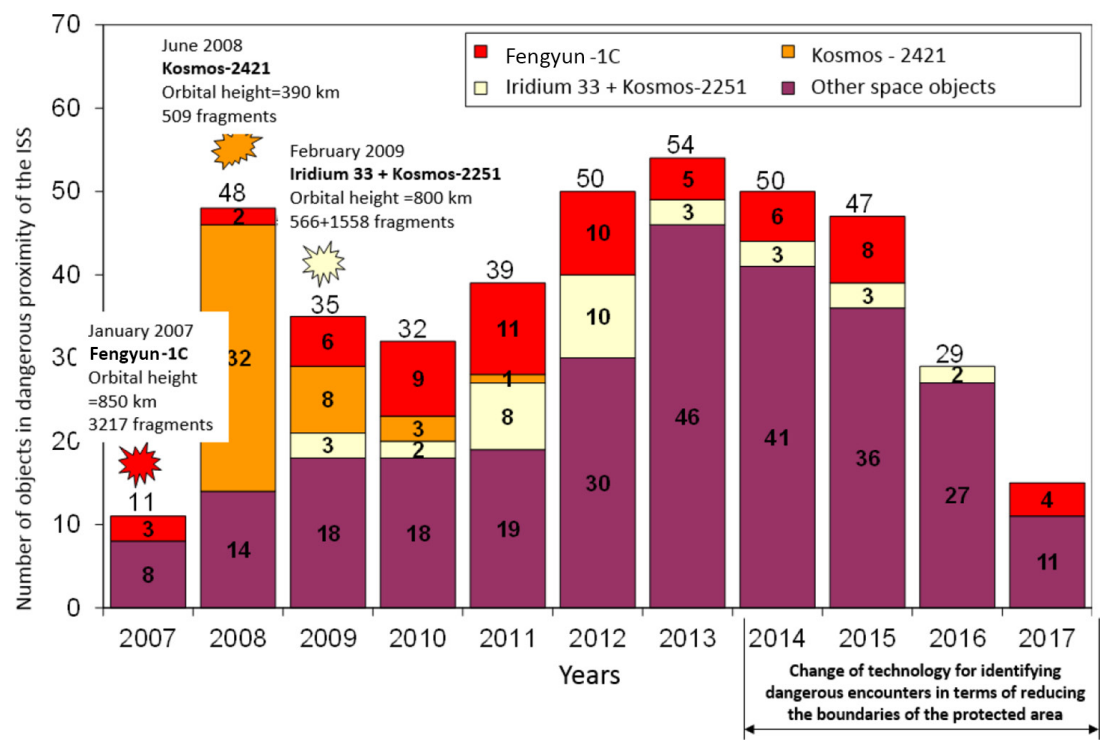

Figure 1: Number of dangerous encounters of the ISS with space debris

hazardous proximity of the spacecraft Kanopus- $V$ with space debris: implementation of evasive maneuver plan required a whole range of activities and involved a whole number of additional specialists from related teams.

At the same time, the number of dangerous encounters with space debris continues to increase (Fig. 1) - a slight decrease in the right side of the chart is due only to the change of rules for registering such encounters.

Technogenic clogging of outer space is one of the most important problems of safe control of spacecraft flight.

If we consider distribution of large-scale space objects over the apogee altitudes and inclinations of their orbits, it is interesting to note that the most intensively clogged near-Earth space regions are in the range of altitudes from $800 \mathrm{~km}$ to $900 \mathrm{~km}$ and inclinations of $70^{\circ}$ to $100^{\circ}$. Cases of destruction of spacecrafts as a result of collision with space debris, as well as ground impact of massive fragments have been recorded. The total mass of space debris in the near-Earth space is currently estimated at around 7,000 tons.

In Russia, automated warning system for dangerous situations in the near-Earth space (AWSDS NES / ASPOS OKP) has been created and is currently in operation. The system includes the main analytical center for monitoring dangerous situations in various areas of the near-Earth space, computation of parameters of solar and geomagnetic activity and analysis of problem situations about space objects [3].

The main tasks of the system are the following:

- continuous monitoring of the technogenic situation in the near-Earth space;

- identification, prediction and issuance of warnings about dangerous approaches of controlled spacecrafts with fragments of space debris, development of recommendations for conducting evasive maneuvers;

- forecasting the entry of uncontrolled risk objects into the atmosphere, determining the coordinates and time of their ground impact; 
- analytical support of concerned parties with data on the technogenic situation;

- implementation of international obligations of the Russian Federation on problems related to space debris.

Systems like the Russian AWSDS NES are already one of the key elements in securing the safety of space flights in the near-Earth space and in the future the role of such systems will only increase.

Another serious challenge for developing control systems for future space systems is dramatic increase in the number of spacecrafts in the orbital space system. Thus, at present, orbital systems have several spacecrafts in their composition, and this is considered the norm. Orbital systems, consisting of several dozen spacecrafts, will be developed as a large space systems in nearest future including hundreds and thousands of spacecrafts. For example, a number of projects for providing global access to broadband Internet with the help of small spacecraft groups are now at various stages of implementation: OneWeb - 720 spacecrafts (+1972 spacecrafts in the long term), SpaceX - 4425 spacecrafts, Boeing - 2956 spacecrafts.

The quantitative transition from dozens to thousands of spacecrafts in space systems will undoubtedly bring the shift of paradigm in operational flight control and significantly influence both the methods and tools for operational management of the space system itself, as well as the types of coordination and interaction of various space systems [4].

Another potentially significant problem is the expansion of the use of nuclear energy in space and, consequently, collisions between space objects with nuclear sources on board and with space debris.

The beginning of the use of nuclear energy in space was marked on June 29, 1961 with the launch of the American spacecraft Transit 4A with the radioisotope thermoelectric generator SNAP-3B. Nuclear fuel, for example, plutonium-238, has long half-life (89 years) and relatively high energy release, which makes it very valuable for space energy.

One of the fundamental guidelines for the use of nuclear energy in space is that the organizations responsible for developing and launching spacecrafts with nuclear sources on board must make every effort to protect people and the biosphere from radiological hazards.

\section{THE NETWORK APPROACH TO SPACECRAFT FLIGHT CONTROL: FROM CONFLICTS TO CONSENSUS}

At present, space systems, as a rule, are de-facto created on the basis of a certain model of flight control. This model describes flight control as the process of changes in the parameters of the spacecraft state, aimed at achieving a given flight goal. Changes in the state parameters include a series of control operations aimed at changing the state of the spacecraft in accordance with the flight plan. The efficiency of such management requires to implement the set of operations in real and/or near-real time [5].

In the communication sessions with the spacecraft the flight control group continuously evaluates the state of the spacecraft. Based on the results of the assessment, a decision is made on the regular or abnormal condition of the spacecraft [6]. If the state is normal, control actions are taken to transfer the spacecraft to another state in accordance with the flight plan. However, if the state is abnormal, there starts the process of developing recommendations and such control actions that would allow the spacecraft to return to the planned state or, at least, to some state that meets the safety requirements of flight control. Such operational 
management implies successive execution of flight control stages: planning, implementation of the plan, monitoring and analysis of results, decision-making on control results.

Traditionally the control system is based on the feedback model, in which it is required to identify the state of control object, decide what actions to take, communicate actions with object and get feedback - to adjust control actions.

As a consequence of using this model in design of control solutions for space systems, in terms of flight control, the following features are required:

- methods and rules of flight control for each spacecraft;

- astro-ballistic models of spacecraft motion;

- specialized software for each spacecraft or space system;

- planning systems;

- integrated analysis of flight control processes and results.

However, with the beginning of mass exploitation of multi-satellite systems in conditions of the growing volume of space debris, the structures and processes arising during the flight control of various spacecraft groups are becoming increasingly connected with each other in a complex manner. Since these links are also able to dynamically change over time, specification of the flight control systems in the form of a classical control models with feedback ceases to satisfy practical requests.

It seems more preferable to have distributed network-based control processes of a satellite group as a whole [7], in which the network of earth-based users, ground and space stations and spacecrafts meet all needs (Fig. 2):

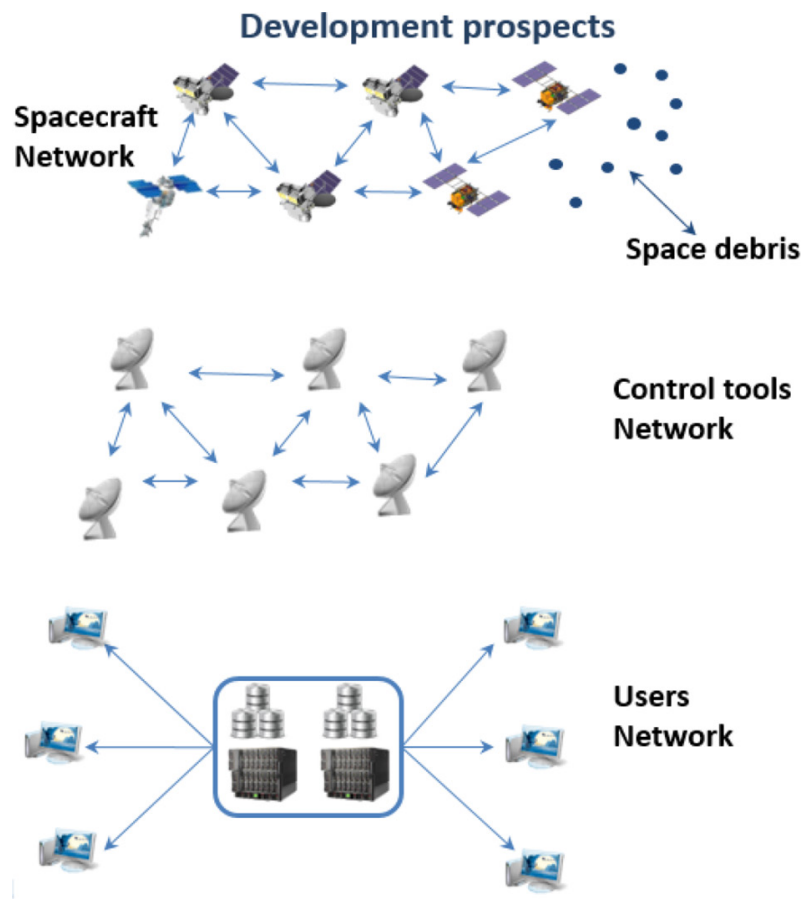

Figure 2: Network model of spacecraft control 
1. Earth-based users have the opportunity to interact with each other, inform each other about their decisions, cooperate and coordinate with each other in achieving their goals, negotiate and compete with each other in the open information field.

2. The number, composition and use of ground and space flight control tools can be dynamically changed depending on the needs and capabilities of earth-based users, as well as on external causes (accidents, malfunctions, in-house maintenance operations, etc.)

3. The number and composition of the spacecraft group is dynamically changed due to entry of new spacecrafts and disposal of spacecrafts that have served their time or due to the consequences of emergency situations.

The use of a "networked" flight control model allows to formulate, at least at the conceptual level, the business requirements of a certain systems which will be a higher-order system (system of systems) in relation to the flight control system of a particular individual spacecraft. The objective of this system should be moderation of the spacecraft network, the network of space stations and management systems and assistance to earth-based users in developing effective interaction and negotiation processes [8].

The proposed network of systems will require the new adaptive resource management technology for organizing and coordinating space traffic [9].

Part of this technology could be blockchain for sharing resources "on demand" between different organizations (for example, ground stations).

\section{DIGITAL PLATFORM AND ECO-SYSTEM OF SMART SERVICES FOR ADVANCED SPACE TRAFFIC MANAGEMENT}

Advanced space traffic management (ASTM) involves digital platform for building an eco-system of smart services $[10,11]$ - to combine different models and methods for developing new generation of space systems.

The working paper submitted by the Russian Federation at the 59th session of the Committee on the Peaceful Uses of Outer Space (30.01.2017 - 02.10.2017), titled "Additional ideas on the set of goals to achieve Vienna consensus on safety in outer space and the need for thorough understanding on the modalities of consideration of complex issues related to space traffic management, and justification of the great expectations of early decisions in this field" may form a new legal basis for developing new space platform for cooperation, suggesting the following steps:

- to create and agree the concept of traffic management in space based on thorough study of all problematic issues and economic risks;

- step transition from ensuring safety of space operations at the level of documents of voluntary execution to the rules governing traffic in space;

- to create a single UN-based digital platform for monitoring and dissemination of information about objects and events in the near-Earth space;

- introduction of formal requirements for accuracy, completeness and timeliness of providing monitoring information about objects and events in the near-Earth space.

The main tasks of creating a digital platform for managing space traffic are the following:

- developing guidelines, standards and procedures relating to safety of space operations and avoiding collision of spacecraft with operating spacecrafts or elements of space debris; 
- development and implementation of rules for provision and use of information on the situation in space;

- prevention of further generation of space debris and implementation of measures for safe cleaning of the near-Earth space from space debris;

- control of the state of the orbital group in order to identify dangerous flight situations and develop recommendations for their parrying in operational mode;

- provision of continuous updating of the space-time model of the near-Earth space;

- development of recommendations on planning of orbital operations in order to improve safety of space systems traffic and movement of individual spacecrafts in the current and forecested space environment;

- timely, complete, authentic and accurate communication of information about objects and events in the near-Earth space to consumers;

- carrying out analysis of processes of managing spacecrafts as a part of space traffic control technology;

- participation in planning and preparation for the launch of new spacecrafts and space systems in order to assess the risks for the expected period of operation, justification of acceptable orbits, correction of time for the launch of carrier rockets and boosters.

Based on these tasks, the following key "smart services" can be developed (Fig. 3):

- smart services for ballistic models;

- smart services for models and methods of data analysis;

- smart services for planning at all levels.

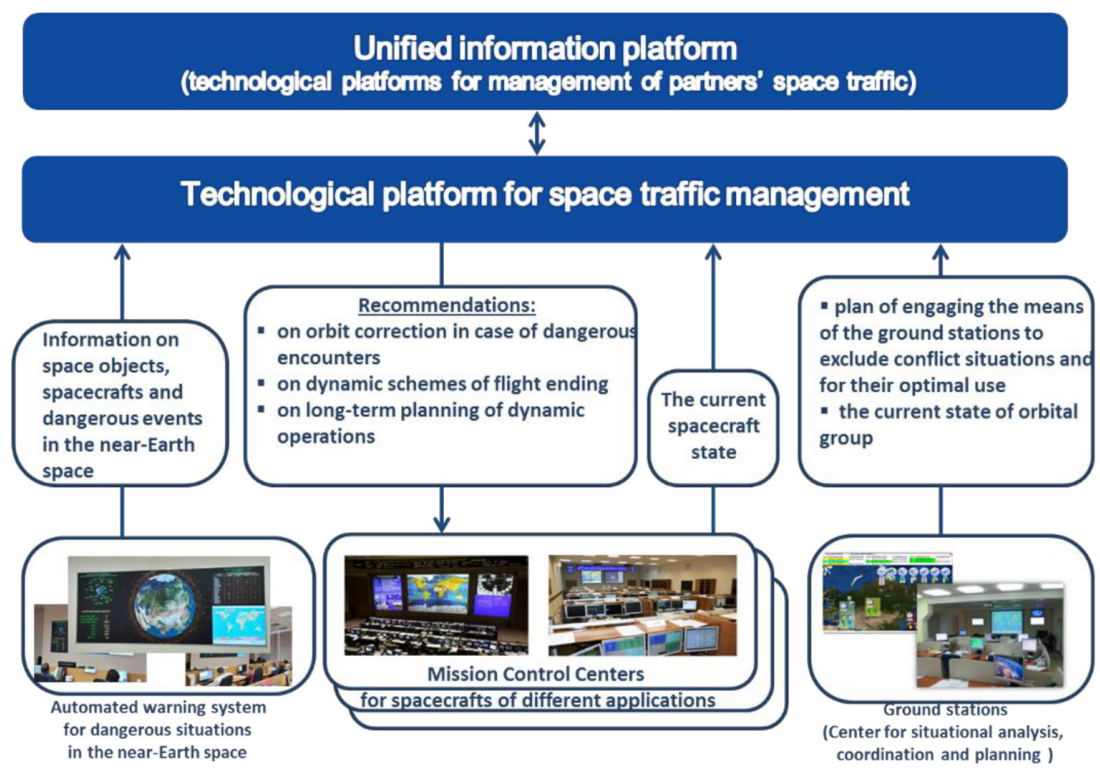

Figure 3: Structure of the digital platform of space traffic management 
The features of any "smart service" should be the following:

- Ontology-driven Knowledge base - which should contain a semantic network of concepts and relations of the domain area [12];

- Autonomy - the ability to continuously respond to events, make plans and achieve their fulfilment.

The platform will be based on mathematical models and methods of ballistics, which should provide the following possibilities:

- creation of a ballistics-navigation support system for flight control for a large number of multi-type spacecrafts controlled from a single mission control center;

- methods of project-ballistic analysis and expertise of orbital construction of space remote sensing systems, communication and retransmission;

- development of software for selection and calculation of parameters of orbits for disposal of spacecrafts;

- improving methods for calculating dangerous encounters and parameters of evasive maneuvers for spacecrafts;

- methods of ballistic design of space complexes for orbiting maintenance of spacecrafts in the near-Earth orbits;

- improvement of methods for calculating ballistic schemes for removal of largesized space debris;

- comprehensive methodology for predicting technogenic pollution of an area of 1100 (up to 2000) km with objects larger than $10 \mathrm{~cm}$;

- development of a methodological approach to assessment of safety of payload generation scheme;

- improvement of methods and technologies for traffic control and prediction of dangerous encounters;

- development of methods and technologies improving the accuracy of forecasting the time and impact point of unmanaged space objects;

- study of the evolution of "clouds" of space debris, formed as a result of destruction of rocket and space equipment objects in the low-earth orbit;

- improving interaction between organizations engaged in safety analysis and preparing and launching space-mission vehicles;

- creation of space observing devices in the visible and infrared ranges for fragments of space debris in the low-earth orbit;

- measures for prevention of the risk of collisions of functioning spacecrafts with formed fragments.

The development of the platform will make possible to significantly improve the quality and efficiency of space traffic management in the context of constantly growing technogenic pollution of the near-Earth space, namely:

- maintenance of space vehicles of the Russian orbital group, as well as identified potentially dangerous space objects and risk objects;

- control of implementation of the stages of spacecraft launch into target orbits, measures to remove the spent Russian spacecrafts, the upper stages of launch ve- 
hicles and boosters into the "dead" zones and into orbits with a limited life, assess parameters of the state of accompanied spacecrafts (including in non-emergency situations);

- assessment, identification, forecast, analysis, and ballistic support of dangerous situations, including destruction of space objects in the near-Earth space, dangerous encounters of spacecrafts with potentially dangerous space objects, forecasting descent from orbits and ground impact of risk objects identifying time and possible impact area.

The next key element of the space traffic management is the data analysis services - aimed to improve technologies for analyzing flights parameters of spacecrafts and their control systems, forecast their functioning on the basis of accumulated knowledge base of flight data, as well as monitor the state of elements of the ground stations.

Figure 4 shows the structure and integration of these services.

The main functions of the data analysis services are the following:

- collection, processing and storage of information on flight data of individual spacecrafts and spacecraft systems;

- forecast of operation of spacecrafts and space systems based on the accumulated knowledge base of flight data;

- monitoring the state of the elements of the ground stations.

The proposed approach provide solution for solving the problems of telemetric and information support for flight control of manned and automatic spacecrafts and space systems for the benefit of creating the unified space traffic management technologyfor a large number of spacecrafts.

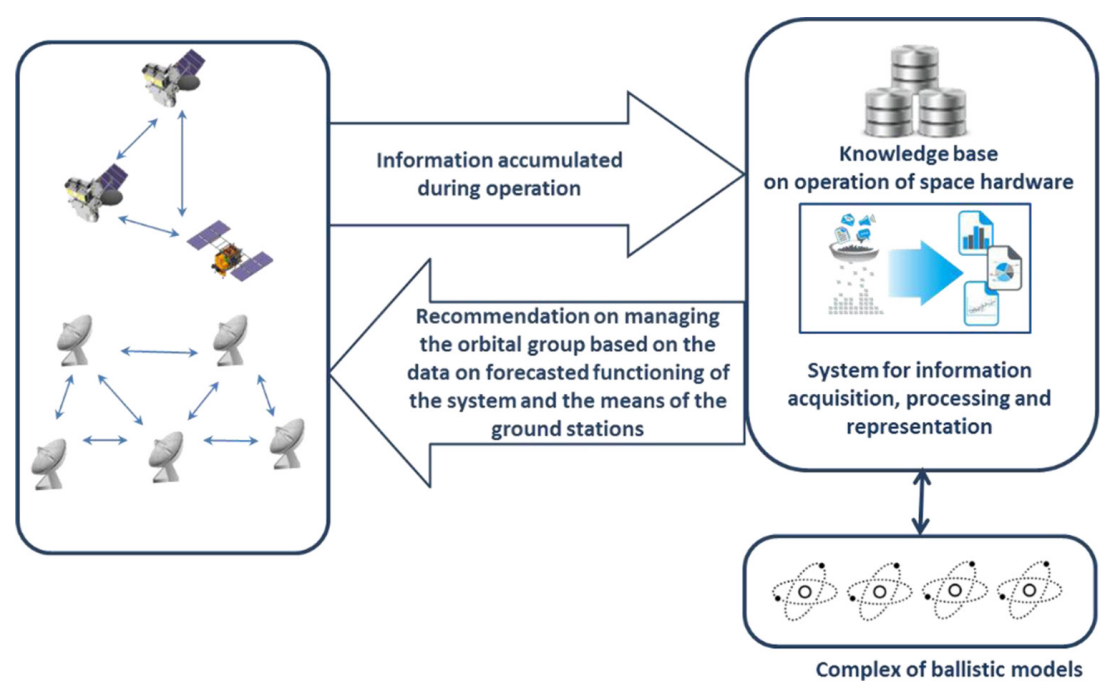

Figure 4: The structure and integration of data analysis services 


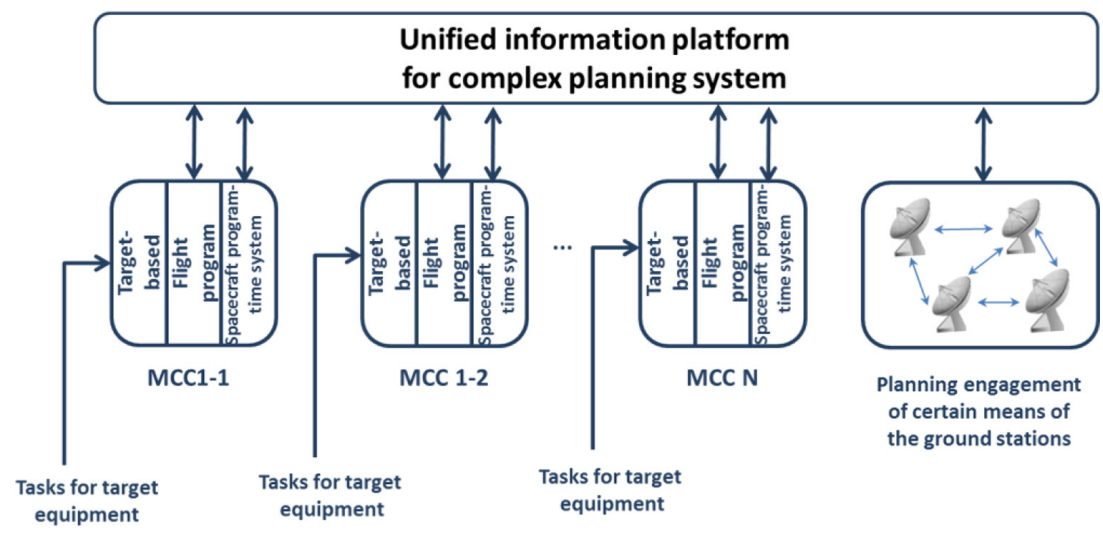

Figure 5: Planning services based on the digital platform

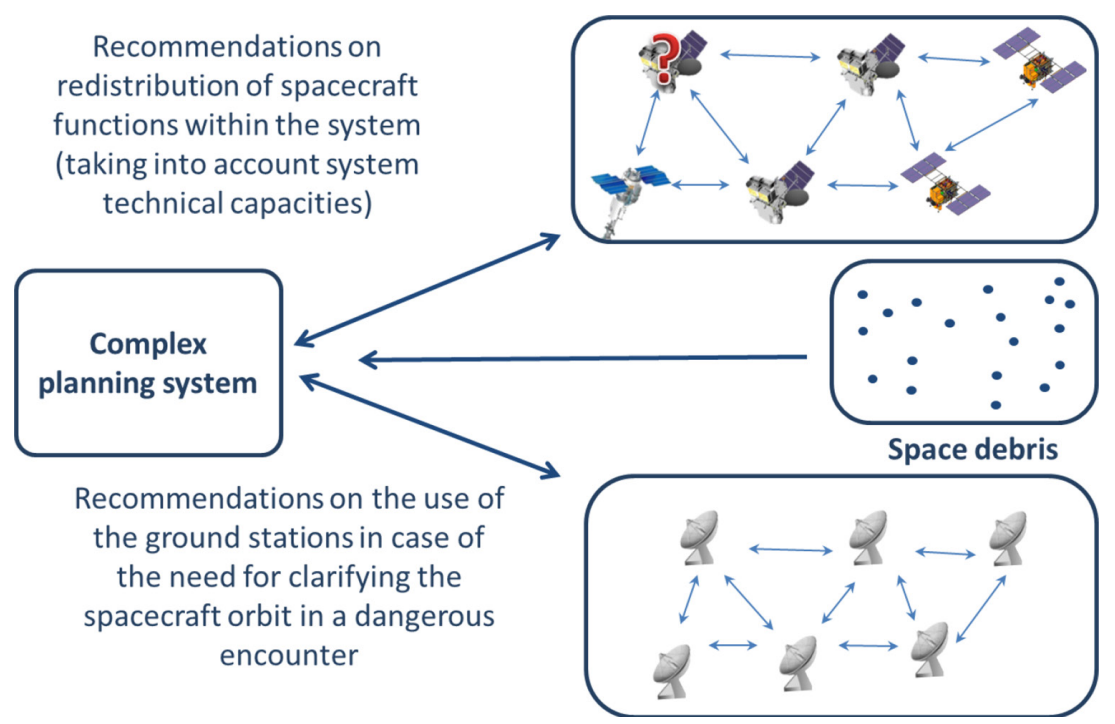

Figure 6: Scheme of task redistribution in case of the event of an abnormal situation

In order to create services for strategic and operational management of large-scale orbital groups of spacecrafts, it is necessary to use models and methods for allocating resources of ground-based and satellite spacecraft control tools, as well as mutual maneuvering of spacecrafts within the existing space technology capabilities (Fig. 5).

With the use of developed platform it becomes possible to provide modernization of the Center for situational analysis, coordination and planning to manage a large number of different types of spacecrafts, as well as to support interaction between spacecrafts and ground stations for scientific, social and economic applications.

At the same time, an end-to-end network of spacecrafts and stations can be provided for managing abnormal situations (Fig. 6). 
In addition, within the platform, there should be implemented services for target planning for groups of various types of spacecrafts, services for mamaging of the spacecraft flight program, services for space-time control of spacecrafts, services for planning the use of individual elements of the ground stations and services for forming tasks for the target equipment. The platform should support organization of tasks redistribution in the spacecraft system in cases of unpredictable events of an abnormal situation and the need for unscheduled evasion from space debris. At the same time, additional services should be implemented for issuing recommendations on redistribution of spacecraft functions within the system (taking into account the technical capabilities of the system) and for issuing recommendations on the use of the ground stations for clarifying the spacecraft orbit in case of a dangerous encounter.

Another important issue in developments of the space traffic management is legal and regulatory framework. Since the space traffic management technology affects aspects of international cooperation, it is necessary to take into account not only the Russian GOST (State All-Union standard), but also international ISO, CCSDS standards, and documents of the Inter-Agency Space Debris Coordination Committee (IADC).

\section{CONCLUSION}

The objective of the developed approach is to create new opportunities for managing space traffic and resources of the mission control centers for a large number of spacecrafts with different owners.

The proposed space traffic management platform and eco-system of smart services will provide a comprehensive solution of the following tasks:

- continuous round-the-clock control of automatic spacecrafts in various orbits;

- cataloguing information on space debris in the near-Earth space;

- rapid identification of dangerous encounters;

- determination of the time and area of ground impact for space objects;

- coordinating the work planning for the ground stations and for spacecrafts of scientific, social and economic applications in order to exclude conflict situations and their optimal use;

- analysis of the use of the ground automated control complex for spacecrafts of scientific, social and economic applications;

- analysis of the status of the orbital group of spacecrafts of scientific, social and economic applications;

- provision of round-the-clock continuous control of the Russian Segment of the ISS RS;

- providing control of Transport Cargo Vehicles of the "Progress MS" and "Soyuz MS" types;

- ensuring the conduct of scientific experiments.

Currently, methods and tools for developing digital platform for control spacecrafts and and use of ground stations are under development.

The main requirement is to manage large-scale orbital groups of up to 40 spacecrafts at the existing ground stations including small, ultra-small and nano spacecrafts that will be formed in a self-organizing "swarm" or "cloud" -to make the Roskosmos orbital group more smart, open and flexible, scalable, efficient and reliable and also protect the spacecrafts from space waste and debris. 


\section{ACKNOWLEDGEMENTS}

The paper has been prepared based on the materials of scientific research within the subsidized state task of the Institute for Control of Complex Systems of the Russian Academy of Sciences for research and development on the topic: "Development of models, methods and tools for supporting and developing mechanisms of multi-level self-organization for improving task and resource management in multi-discipline projects of manufacturing complex technical products".

\section{REFERENCES}

[1] Lobachev, V.I. \& Pochukaev, V.N., "Methods of testing rocket and space equipment", Mechanical Engineering, 1995. Section "Means of flight control of scientific and manned space vehicles".

[2] Matyushin, M.M., Sokolov, N.L. \& Zakharov, P.A., Features of control of large-scale groups of space vehicles. Problems of Control and Modeling in Complex Systems, 2016.

[3] Stepanov, I.B. \& Pavlova, S.A., Events in near-Earth space // TsNIIMash, Information and Analytical Support of ASPOS OKP (automated warning system for dangerous situations in the near-Earth space). The Main Information and Analytical Center of ASPOS $O K P, \mathbf{9}(\mathbf{7 6}), 48 \mathrm{p}$.

[4] Udaloy, V.A. \& Sokolov, N.L., On the possibility of mathematical formalization of the functioning processes of the Mission Control Center as a large control system. Modern Science-Intensive Technologies, 2006.

[5] Matyushin, M.M., Sokolov, N.L. \& Ovechko, V.M., Methodological approaches to solving problems of managing large-scale groups. Engineering Journal: Science and Innovation, 51, 2016.

[6] Matyushin, M.M., Potockiy, S.I., Skobelev, P.O., Potapov, V.I. \& Lakhin, O.I., Automated decision-support system for emergency situations. Software Products and Systems, (3), pp. 62-69, 2013.

[7] Matyushin, M.M., Sokolov, N.L., Ovechko, V.M. \& Polivnikov, V.M., Designing rational schemes for distribution of control means during operation of large-scale orbital groups of space vehicles. Flight. All-Russian Scientific and Technical Journal, 2016.

[8] Rzevski, G. \& Skobelev, P., Managing Complexity. Southampton, Boston, WIT Press, 2014.

[9] Skobelev, P.O., Lakhin, O.I., Polnikov, A.S. \& Simonova, E.V., Approach to the solution of aerospace product lifecycle management problem based on network-centric principles. Proceedings of the 7th International Conference on Industrial Applications of Holonic and Multi-Agent Systems (HoloMAS'2015), eds. V. Marik, J.I. et al., , Valencia, Spain. - HoloMas 2015, pp. 169-178, September 2-4, 2015.

[10] Digital ecosystem, avialbale at https://en.wikipedia.org/wiki/Digital_ecosystem (accessed 18 March, 2018).

[11] Skobelev, P., Multi-agent systems for real time adaptive resource management. Industrial Agents: Emerging Applications of Software Agents .in Industry, ed. Paulo Leitão \& Stamatis Karnouskos. Elsevier, pp. 207-230, 2015.

[12] Skobelev, P., Activity ontology for situational management of enterprises in real-time. Ontology of Designing, 1(3), pp. 6-38, 2012. 